\title{
Analytical Aberration-Corrected Electron Microscopy Study of the Effects of Transient Operation on the Stability of Nanostructured Thin Film Catalysts for PEM Fuel Cells
}

D.A. Cullen, ${ }^{*}$ K.L. More, ${ }^{*}$ K.S. Reeves, ${ }^{*}$ G.D. Vernstrom, ${ }^{* *}$ L.L. Atanasoska, ${ }^{* *}$ G.M. Haugen, ${ }^{* *}$ and R.T. Atanasoski**

* Materials Science \& Technology Division, Oak Ridge National Laboratory, Oak Ridge, TN 37831

** 3M Fuel Cell Components, 3M Central Research, St. Paul, MN 55144

Improving the durability and stability of materials comprising proton exchange membrane fuel cells (PEMFCs) is a critical milestone in the development of these systems for efficient and cost-effective power generation [1]. A primary concern is the durability of catalysts during transient operation, which can occur during startup/shutdown or under $\mathrm{H}_{2}$-fuel starvation when the cathode is subjected to high potentials $(>1.2 \mathrm{~V})$. In terms of catalyst utilization, maximizing and maintaining the electrochemical surface area of expensive transition metals, in particular Pt, is key to producing a durable and cost-effective technology.

Nanostructured thin films (NSTFs) are a novel and attractive support for Pt and Pt-alloy catalysts. The thin film, continuous catalysts are supported on vertically aligned, crystalline, organic whiskers [2], and membrane electrode assemblies (MEAs) built around this system have been reported to improve oxygen reduction activity by more than $10 \mathrm{X}$ compared with conventional carbon-supported electrocatalysts [3]. In order to preserve this excellent activity during exposure to transient operating conditions, extremely small amounts of Ir and $\mathrm{Ru}$ are incorporated in the catalyst surfaces. The role of these elements is to decrease the high electrode potentials by electrolyzing water more efficiently than Pt.

The high spatial resolution of an aberration-corrected JEOL 2200FS scanning transmission electron microscope (STEM), equipped with a Bruker X-Flash Si drift detector (SDD) for energy dispersive spectroscopy (EDS), was used to elucidate the microstructural causes of catalyst deactivation in $\mathrm{Ru}-\mathrm{Ir}-\mathrm{Pt}$ NSTFs. Fig. 1 shows SEM and STEM images of the untested NSTF. EDS elemental mapping of individual whiskers confirmed the presence of Ir and Ru on the Pt-catalyst whisker surfaces, and imaging of the whisker edges showed Ir and Ru often took the form of small nanoparticles (Fig. 2). These NSTFs were assembled into an MEA and exposed to electrode potentials up to $2 \mathrm{~V}$. EDS elemental maps of the tested cathode NSTF show a significant loss of Ir and Pt from the NSTF surfaces due to $\mathrm{Pt}^{2+}$ and $\mathrm{Ir}^{2+}$ migration from the cathode into the membrane. Individual Pt-Ir nanoparticles form within the membrane close to the cathode as a result of this ionic migration, and larger Pt agglomerates are found deeper in the membrane (Fig. 3) [4]. Ir did not diffuse as readily as $\mathrm{Pt}$, nor did the Ir form large agglomerated particles. The concentration of any remaining $\mathrm{Ru}$ was too low to be detected by EDS.

\section{References}

[1] Y. Shao, G. Yin, and Y. Gao, J. Power Sources 171 (2007) 558.

[2] M.K. Debe and A.R. Drube, J. Vac. Sci. Technol. B 13 (1995) 1236.

[3] L. Gancs et al., Chem. Mater. 20 (2008) 2444.

[4] R.M. Darling and J.P. Meyers, J. Electrochem. Soc. 150 (2003) A1523.

[5] This work was supported by (1) the Fuel Cell Technologies Program, Office of Energy Efficiency and Renewable Energy, U.S. Department of Energy under Award Number DE-EE0000456 and (2) ORNL's SHaRE User Facility, Office of Basic Energy Sciences, U.S. Department of Energy. 

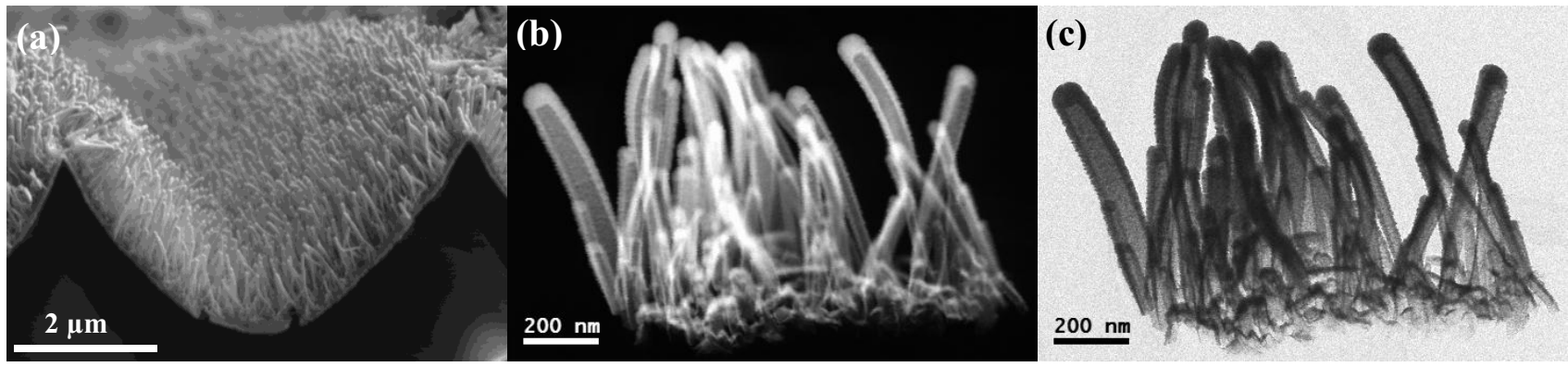

FIG. 1. (a) SEM image of catalyst coated crystalline organic whiskers (NSTF). (b) Dark-field (DF) and (c) bright-field (BF) STEM images of untested Pt+RuIr coated NSTF.
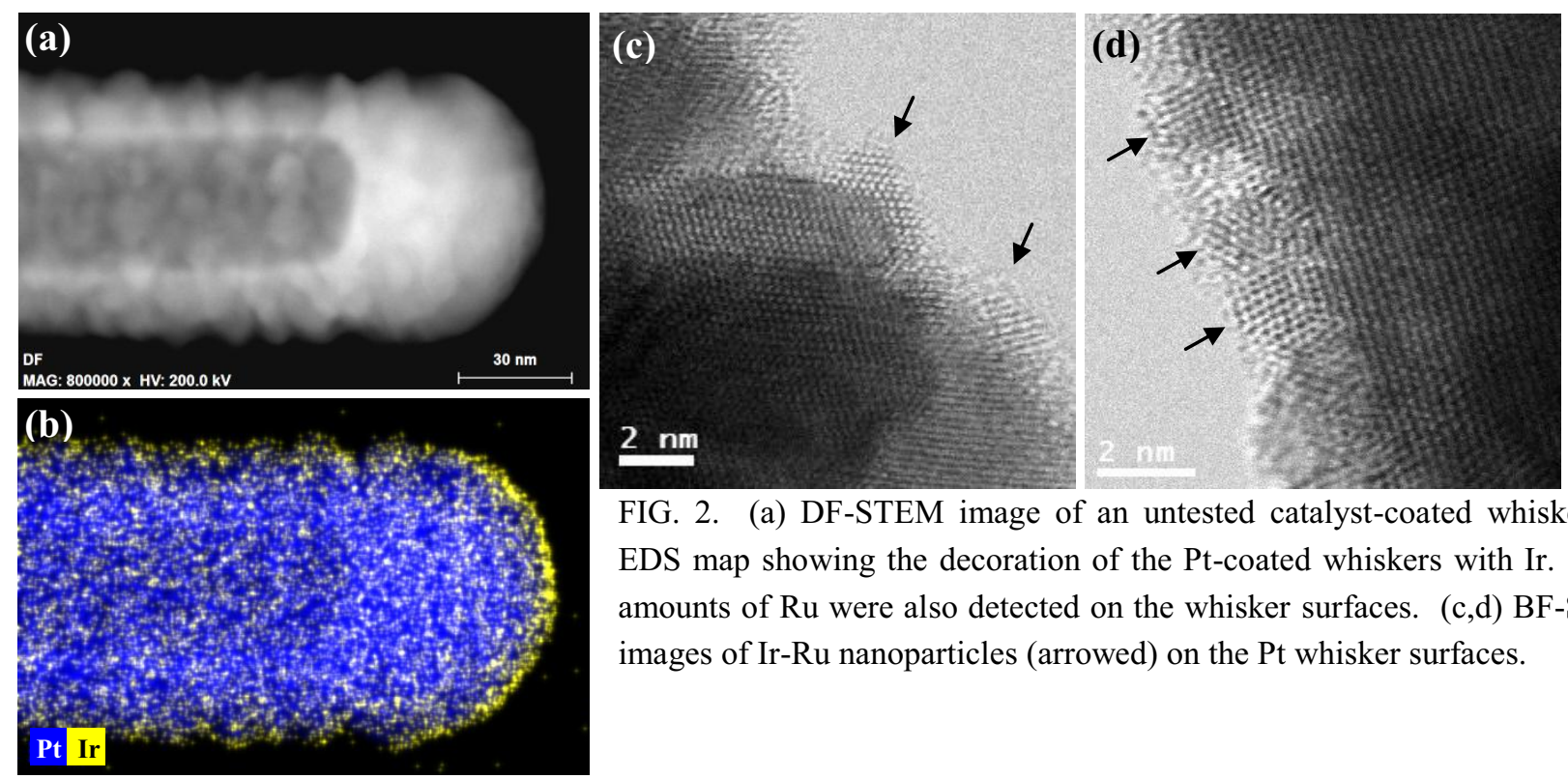

FIG. 2. (a) DF-STEM image of an untested catalyst-coated whisker. (b) EDS map showing the decoration of the Pt-coated whiskers with Ir. Trace amounts of Ru were also detected on the whisker surfaces. (c,d) BF-STEM images of Ir-Ru nanoparticles (arrowed) on the Pt whisker surfaces.
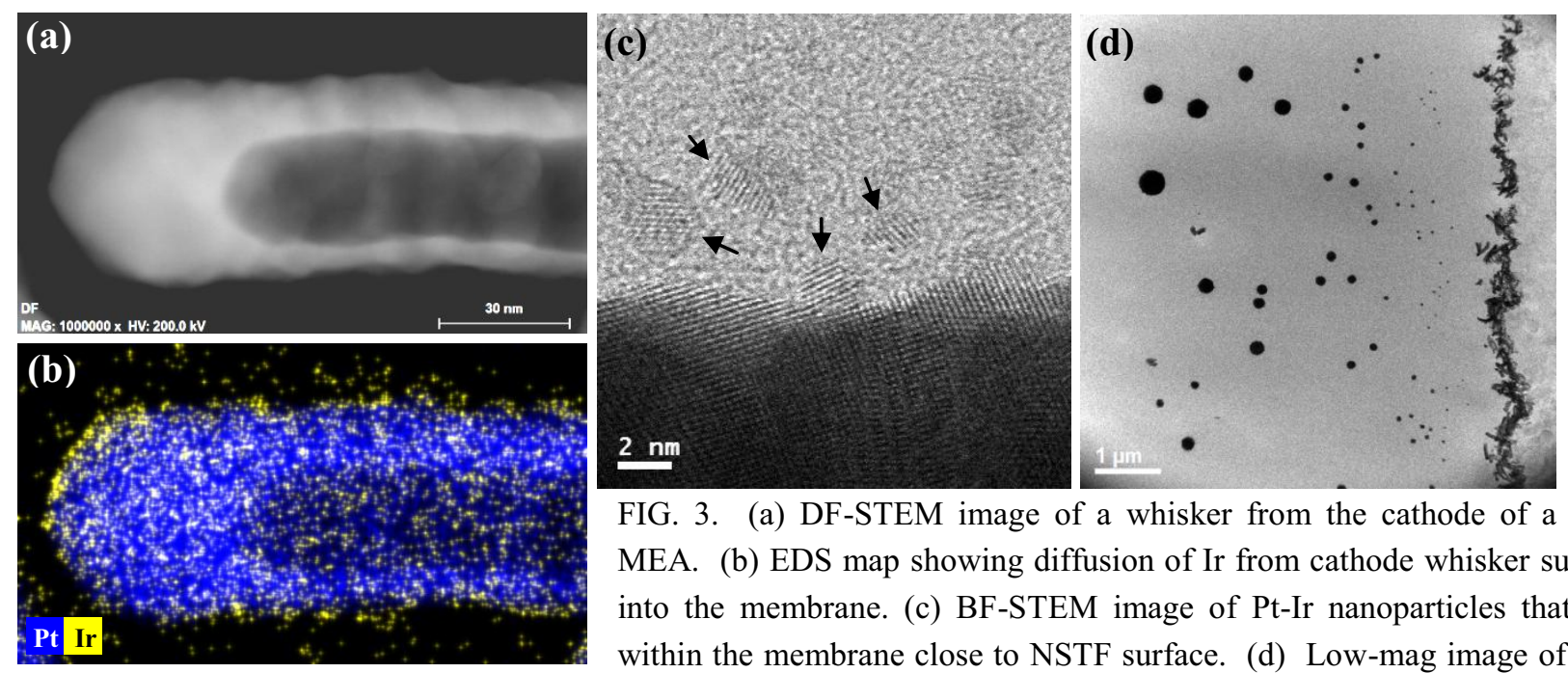

FIG. 3. (a) DF-STEM image of a whisker from the cathode of a tested MEA. (b) EDS map showing diffusion of Ir from cathode whisker surfaces into the membrane. (c) BF-STEM image of Pt-Ir nanoparticles that form within the membrane close to NSTF surface. (d) Low-mag image of MEA cathode, showing the formation of large Pt particle agglomeratess deep in the polymer membrane. 